CLINICAL STUDY

\title{
Addison's disease: a survey on 633 patients in Padova
}

Corrado Betterle, Riccardo Scarpa, Silvia Garelli, Luca Morlin, Francesca Lazzarotto, Fabio Presotto ${ }^{1}$, Graziella Coco ${ }^{\dagger}$, Stefano Masiero, Anna Parolo, Maria Paola Albergoni ${ }^{2}$, Roberta Favero ${ }^{2}$, Susi Barollo, Monica Salvà, Daniela Basso ${ }^{3}$, Shu Chen ${ }^{4}$, Bernard Rees Smith ${ }^{4}$, Jadwiga Furmaniak ${ }^{4}$ and Franco Mantero Endocrine Unit, Department of Medicine, University of Padova, Via Ospedale Civile, 105, 35128 Padova, Italy, ${ }^{1}$ Unit of Internal Medicine, General Hospital of Mestre, Venice, Italy, ${ }^{2}$ Blood Transfusion Center, Azienda Ospedaliera-Universitaria of Padova, Padova, Italy, ${ }^{3}$ Department of Laboratory Medicine, University of Padova, Padova, Italy and ${ }^{4}$ FIRS Laboratories RSR Ltd., Cardiff, UK

(Correspondence should be addressed to C Betterle; Email: corrado.betterle@unipd.it)

${ }^{\dagger}(\mathrm{G}$ Coco is deceased)

\begin{abstract}
Objective: Addison's disease (AD) is a rare endocrine condition.

Design: We aimed to evaluate clinical, immunologic, adrenal imaging, and genetic features in 633 Italian patients with AD followed up since 1967.

Methods: Adrenal cortex autoantibodies, presence of other autoimmune and nonautoimmune diseases, nonadrenal autoantibodies, adrenal imaging, and genetic profile for HLA-DRB1 and AIRE were analyzed.

Results: A total of 492 (77.7\%) patients were found to be affected by autoimmune AD (A-AD), 57 (9\%) tuberculous AD, 29 (4.6\%) genetic-associated AD, 10 (1.6\%) adrenal cancer, six (0.94\%) post-surgical $\mathrm{AD}$, four $(0.6 \%)$ vascular disorder-related $\mathrm{AD}$, three $(0.5 \%)$ post-infectious $\mathrm{AD}$, and $32(5.1 \%)$ were defined as idiopathic. Adrenal cortex antibodies were detected in the vast majority (88-100\%) of patients with recent onset $\mathrm{A}-\mathrm{AD}$, but in none of those with nonautoimmune AD. Adrenal imaging revealed normal/atrophic glands in all A-AD patients: $88 \%$ of patients with $\mathrm{A}-\mathrm{AD}$ had other clinical or subclinical autoimmune diseases or were positive for nonadrenal autoantibodies.

Based on the coexistence of other autoimmune disorders, $65.6 \%$ of patients with A-AD were found to have type 2 autoimmune polyendocrine syndrome (APS2), 14.4\% have APS1, and 8.5\% have APS4. Class II HLA alleles DRB1*03 and DRB1*04 were increased, and DRB1*01, DRB1*07, DRB1*013 were reduced in APS 2 patients when compared with controls. Of the patients with APS1, 96\% were revealed to have AIRE gene mutations.

Conclusions: A-AD is the most prevalent form of adrenal insufficiency in Italy, and $\sim 90 \%$ of the patients are adrenal autoantibody-positive at the onset. Assessment of patients with A-AD for the presence of other autoimmune diseases should be helpful in monitoring and diagnosing APS types 1, 2, or 4 and improving patients' care.
\end{abstract}

European Journal of Endocrinology 169 773-784

\section{Introduction}

Addison's disease $(\mathrm{AD})$ is a primary adrenal insufficiency resulting from bilateral destruction or dysfunction of the adrenal cortex (1). This is a rare endocrine condition with a prevalence increasing progressively in Europe from 39 cases/million of individuals in 1968 to 144 cases in the recent years $(2,3,4,5,6,7,8)$ and about 50 per million in the USA (9).

With regard to the causes of $\mathrm{AD}$, the adrenalitis due to tuberculosis fell from 79 to $17 \%$ during the years 1930-1974 (2, 3, 10, 11).

Autoimmune AD (A-AD) is currently the most common form of adrenal failure in adults $(1,7,8,12$, $13,14,15)$, while it is less frequent in children where genetic forms, mainly congenital adrenal hyperplasia, are the most common causes $(16,17,18)$.
A-AD is frequently associated with other clinical autoimmune diseases. In a recent study of $48 \mathrm{~A}-\mathrm{AD}$ patients from England, $58 \%$ had associated autoimmune hypothyroidism, $29 \%$ vitamin $\mathrm{B}_{12}$ deficiency, and $10 \%$ type 1 diabetes mellitus (DM1). However, autoantibody screening was not systematically carried out in patients with apparently isolated A-AD (19).

In a nationwide Norwegian study, 426 patients with A-AD were evaluated and associations with one or more clinical autoimmune diseases have been found in $61 \%$ (47\% showed thyroid autoimmune diseases, 12\% DM1, $11 \%$ vitiligo, and $10 \%$ pernicious anemia), while $39 \%$ had clinically isolated A-AD (8). However, $27 \%$ of the patients with isolated A-AD were found to be positive for nonadrenal autoantibodies (8). Based on this approach, $88 \%$ of Norwegian A-AD patients were found to have one or more clinical or subclinical autoimmune disease 
or were positive for nonadrenal autoantibodies, while only $12 \%$ had true isolated $\mathrm{A}-\mathrm{AD}$, i.e. without the evidence of autoimmune responses to other targets (8).

In this study, we retrospectively reviewed 633 Italian patients with $\mathrm{AD}$ registered at our Endocrine Unit and classified them according to etiological, clinical, immunologic, and genetic features.

\section{Patients and methods}

Six-hundred and thirty-three patients with AD were registered and followed up in our Endocrine Unit during the years 1960-2010. Our Endocrine Unit is one of the major centers in Italy for the study of adult AD patients, and the majority of the patients came from Veneto and the Northern regions of Italy. A minor proportion of patients came from the other regions in Italy.

The mean period of follow-up was 10.5 years (range 0.5-43 years). Patients were periodically assessed by clinical examination including specific function tests when appropriate and tested for a wide range of autoantibodies.

Clinical autoimmune disease was defined by the presence of typical clinical signs and symptoms, impaired function tests, and presence of the respective autoantibodies. Patients who were positive for autoantibodies and presented impaired relevant function tests in the absence of symptoms of a clinical disease were classed as having subclinical autoimmune disease.

Patients with the specific organ-related autoantibodies in the absence of detectable impairment of the target organ and a clinical disease were also included in the study $(1,20)$. These patients were included because they are at risk of progression to overt diseases and, thus, periodically monitored and assessed for symptoms of clinical autoimmune diseases.

The study was approved by the Ethical Committee of the Azienda Ospedaliera-Università of Padova and all the patients gave their written informed consent to the study. The study was performed according to principles of the Helsinki Declaration.

For the purpose of this study, $\mathrm{AD}$ was defined by the presence of clinical signs and symptoms of hypoadrenalism with low plasma cortisol and increased ACTH levels. All patients were on substitutive therapy for adrenal insufficiency at the time of the recruitment. Patients with one autoimmune disease and adrenal autoantibodies only and normal plasma renin activity, ACTH, and cortisol levels were excluded from this study.

$\mathrm{A}-\mathrm{AD}$ was defined as above in patients positive for adrenal cortex autoantibodies (ACA) and/or 21-hydroxylase autoantibodies (21-OHAbs) or in their absence when $\mathrm{AD}$ was associated with one or more other nonadrenal autoimmune diseases and/or other autoantibodies with normal/atrophic adrenal glands on imaging.
Patients with thyroid autoimmunity included those with i) chronic thyroiditis (CT; diagnosed by the presence of at least two of the following: thyroid autoantibodies with a titer above the reference range, typical thyroid ultrasound pattern, impaired thyroid function) and ii) active Graves' disease (GD) on treatment with anti-thyroid drugs or in remission with normal thyroid function or patients on thyroxine with hypothyroidism occurring spontaneously or as the result of surgical/radioiodine treatment. In addition, patients with one or more thyroid autoantibodies with normal thyroid function tests were included in the group for the purpose of this study.

Patients with beta-cell autoimmunity had i) DM1 or DM2 with islet cell antibodies (ICA) and/or glutamic acid decarboxylase antibodies (GADAbs) and ii) detectable ICA and/or GADAbs with impaired glucose tolerance. Furthermore, patients with detectable ICA and/or GADAbs and normal glucose tolerance were included.

Patients with gastric autoimmunity were presented with: i) autoimmune gastritis with parietal cell autoantibodies, hypergastrinemia, and microcytic irondeficient anemia; ii) autoimmune gastritis with parietal cell autoantibodies and/or intrinsic factor antibodies, hypergastrinemia, and macrocytic anemia (pernicious anemia); and iii) positive parietal cell antibodies with increased gastrin levels and/or anemia, but without gastroscopic evidence of autoimmune gastritis. Also, patients positive for parietal cell antibodies with either normal gastrin levels and red blood cell count were included.

Patients with ovarian autoimmunity were presented with i) premature ovarian failure (POF) with hypergonadotrophic hypogonadism, autoantibodies to steroid-producing cells (StCA), and/or to $17 \alpha$-hydroxylase (17 $\alpha$-OHAbs) and/or to side-chain cleavage enzyme (SCCAbs) and ii) positive StCA and/or 17 $\alpha$-OHAbs and/or SCCAbs with increased serum gonadotropin, normal estrogens levels, and normal menses. Patients positive for StCA, $17 \alpha$-OHAbs, and SCCAbs with normal menses and normal serum levels of gonadotropins were also included.

Patients with celiac disease were presented with i) positive endomysial antibodies (EmA) or tissue transglutaminase autoantibodies (tTGAbs) and villous atrophy with intraepithelial lymphocytic infiltration of the jejunal mucosa with clinical manifestations (clinical disease) and ii) positive tTGAbs and/or EmA, and evidence of villous atrophy with lymphocytic infiltration of the jejunal mucosa without clinical manifestations. Furthermore, the group included patients with tTGAbs and/or EmA only without clinical manifestations and with normal jejunal histology.

Autoimmune malabsorption was defined by the presence of i) tryptophane hydroxylase autoantibodies (TPHAbs) with gastrointestinal dysfunction and low serum levels of serotonin and ii) TPHAbs without 
gastrointestinal dysfunction, but with low serum levels of serotonin (21). Patients with TPHAbs without gastrointestinal dysfunction and with normal serum levels of serotonin were also included (21).

Other autoimmune diseases (alopecia, vitiligo, myasthenia gravis, Sjogren's syndrome, multiple sclerosis, etc.) were diagnosed on the basis of clinical criteria and by specific laboratory tests.

A-AD patients who had other autoimmune diseases were diagnosed with autoimmune polyendocrine syndrome (APS) according to Neufeld and Blizzard's original criteria (22) and to the modified criteria proposed by Betterle et al. $(1,23)$. Specifically, patients were assigned to APS1 when A-AD was associated with chronic candidiasis (CC) and/or chronic hypoparathyroidism $(\mathrm{CH})$. Patients were classed as having APS2 when A-AD was associated with clinical thyroid and/or beta-cells autoimmunity (complete forms). A-AD patients with incomplete APS2 had the evidence of thyroid and/or beta cells autoimmunity in the absence of clinical diseases. Patients with complete APS4 had A-AD associated with other clinical autoimmune diseases except DM1 or thyroid autoimmunity (complete form), while patients with an incomplete form of APS4 were positive for autoantibodies (except for thyroid and diabetes associated autoantibodies) without overt diseases $(1,23)$.

AD patients with ACA and/or 21-OHAbs without any clinical and subclinical autoimmune diseases or nonadrenal autoantibody positivity were defined as having isolated A-AD.

AD patients without ACA, 21-OHAbs, or any evidence of clinical or subclinical autoimmune diseases, or negative for autoantibodies with normal adrenal imaging were classified as having idiopathic AD.

\section{Autoantibodies}

At the entry into the study, and periodically during the follow-up, 619 out of the 633 patients with AD were tested for ACA and 473 for 21-OHAbs, as previously described $(24,25)$.

Recently, the measurements of 21-OHAbs in our laboratory were assessed in an inter-laboratory concordance study for 21-OHAbs determinations, carried out to compare measurements from different laboratories using different methods (26). This study showed strong concordance among laboratories on reporting 21-OHAbs-positive/-negative samples. The sensitivity for $\mathrm{A}-\mathrm{AD}$ was $>80 \%$ in all the four participating laboratories (26).

Furthermore, irrespective of clinical features, all the patients were screened for autoantibodies to thyroglobulin (TgAbs), thyroid microsomes (TMAbs) or thyroperoxidase (TPOAbs), parietal cells (PCA), intrinsic factor (IFA), ICA, GADAbs, StCA, 17 $\alpha-\mathrm{OHAbs,}$ SCCAbs, melanin-producing cells (MPCAbs), liver-kidney microsomes (LKMAbs), IgA-tTGAbs or $\operatorname{IgA-EmA,~}$
aromatic-L-aminoacid decarboxylase (AADCAbs), and TPHAbs, as previously reported $(27,28,29,30)$.

In patients with APS1 or in those with $\mathrm{AD}$ who developed the disease under the age of 20 years, antibodies to interferon omega (IFNAbs) were tested, as previously described (31).

In patients with nonadrenal autoantibody positivity only (detected before, at the onset, or after the diagnosis of A-AD), the annual incidence of the related disorders (clinical or subclinical according to the criteria defined above) as, clinical or subclinical autoimmune thyroid diseases, autoimmune gastritis, pernicious anemia, DM1, POF or celiac disease, was calculated by dividing the number of newly affected patients by the patients' years of follow-up. Each patient contributed to the total sum of patients' years with a period ranging from the start of the detection of the specific autoantibody until the disease was diagnosed or the observation period ended.

\section{HLA-DRB1 typing}

HLA-DRB1 molecular typing at low resolution level was performed in 229 patients with A-AD: 154 with APS2, 33 with APS1, 31 with isolated A-AD, and 11 with APS4. A total of 300 healthy donors randomly selected from the Bone Marrow Donor Register PD02 were used as controls. DNA was extracted from $400 \mu \mathrm{l}$ of peripheral blood using a QIAamp DNA minikit (Qiagen). HLA typing was carried out using PCR sequencespecific primers (PCR-SSP) using commercial kits (Olerup SSP, Stockholm, Sweden). For each sample, 23 PCRs were performed and the amplification products were detected by electrophoresis on $2 \%$ agarose gel (32).

The phenotypic frequencies were compared with those obtained from the controls and $P$ values were calculated using Fisher's exact test, two tails.

\section{AIRE gene mutations}

All the 14 exons of the AIRE gene and their flanking exon-intron boundaries (GenBank accession no. Aj009610) were analyzed using the methods previously described (33) in 100 patients with A-AD; specifically 70 in the context of APS1 and 30 with other forms of A-AD (APS2, APS4, or isolated disease) diagnosed before 20 years of age. A group of 150 healthy blood donors was used as controls.

\section{Imaging}

Imaging of the adrenal glands using computerized tomography or magnetic resonance imaging was carried out in 317 patients with AD (240 with A-AD, 50 with tuberculous $\mathrm{AD}$ (TBC-AD), ten with cancer (C-AD), 15 with genetic forms (G-AD), one with antiphospholipid syndrome, and one with blastomycosis). 


\section{Treatment}

Different forms of treatment received for adrenal failure were analyzed in 610 of our $\mathrm{AD}$ patients.

\section{Results}

\section{Etiology of AD}

Out of the 633 patients, $492(77.7 \%)$ had A-AD, 57 $(9.0 \%)$ had TBC-AD, 29 (4.6\%) had G-AD, $10(1.6 \%)$ had C-AD, six $(0.95 \%)$ had post-adrenalectomy AD (S-AD), four $(0.6 \%)$ had vascular disorders $(\mathrm{V}-\mathrm{AD})$, in three patients $(0.5 \%) \mathrm{AD}$ resulted from infections with adrenal involvement (I-AD), and $32(5.1 \%)$ were idiopathic.

The frequency of different forms of $\mathrm{AD}$ (A-AD, $\mathrm{TBC}-\mathrm{AD}$, and other forms) diagnosed during the various decades from 1960-1970 to 2000-2012 was summarized in Fig. 1.

\section{Patients with A-AD}

The 492 patients with A-AD were followed up from the onset of the disease for a mean period of 10.5 years (range 0.5-43 years) and periodically assessed for the presence of other autoimmune diseases (clinical or

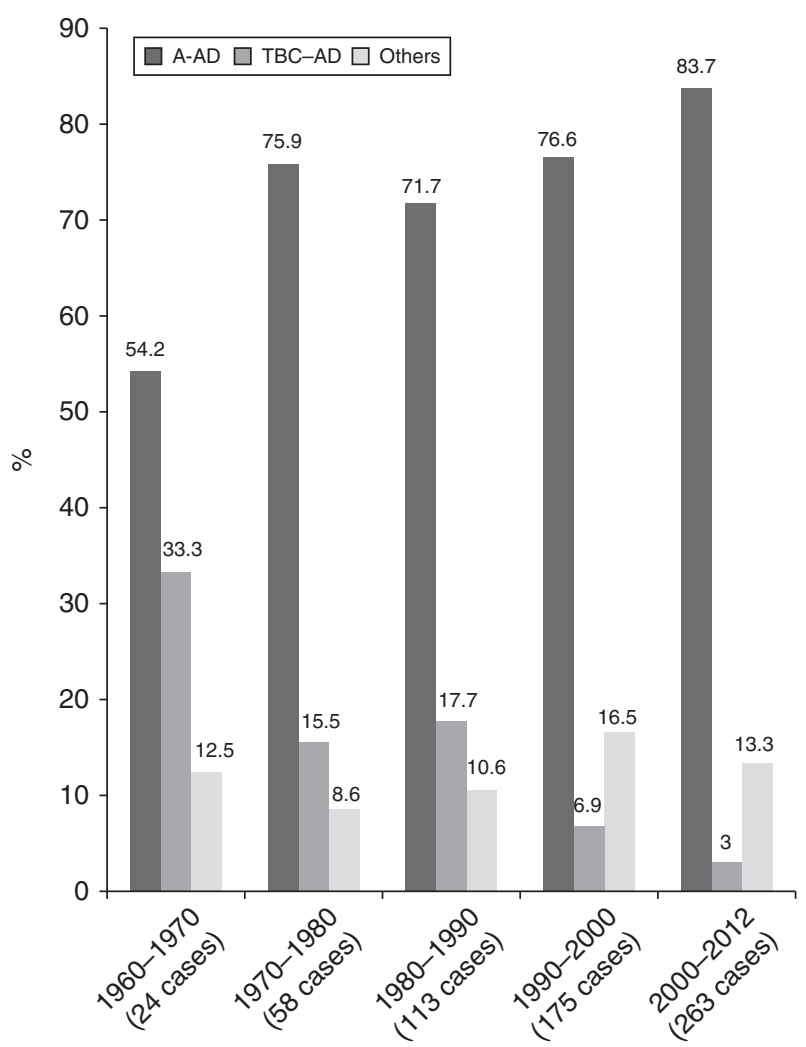

Figure 1 Frequencies of different forms of $A D$, diagnosed during the period from 1960 to 2012 .

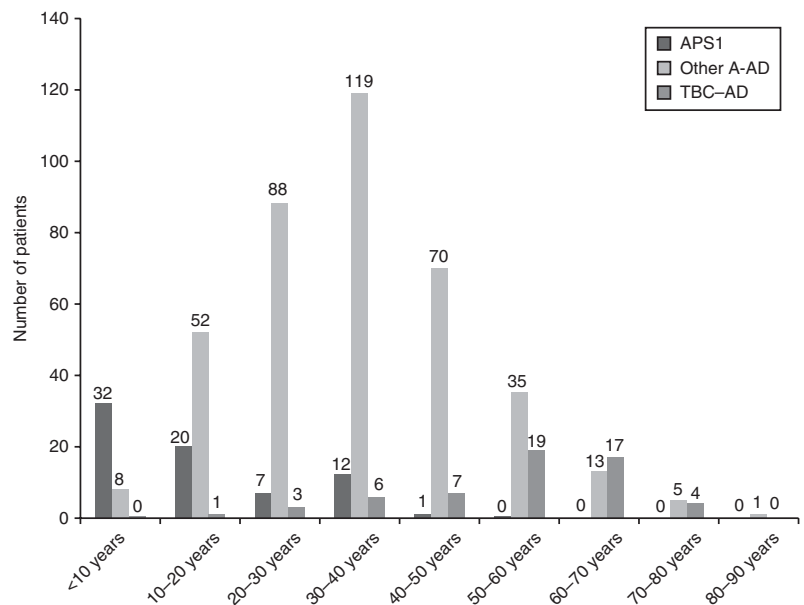

Figure 2 Age at the onset of AD in patients with APS1, other forms of autoimmune AD (APS2, APS4, and isolated A-AD) and with tuberculosis disease (TBC-AD).

subclinical) and/or autoantibody positivity. A-AD patients with associated autoimmune diseases were assigned to different APS according to previously reported criteria (20).

Overall, 436 of 492 patients ( $88.6 \%$ ) were assigned to APS forms. In particular, $322(65.6 \%)$ had APS2, 72 (14.4\%) APS1, 42 (8.5\%) APS4, while $56(11.4 \%)$ had isolated A-AD. For the purpose of this study, patients with APS1, APS2, and APS4 included patients with clinical and subclinical autoimmune diseases and the patients positive for nonadrenal autoantibodies without clinical disease. Figure 2 shows the age at $\mathrm{AD}$ onset in patients with APS1, with other forms of A-AD, or in those with $\mathrm{TBC}-\mathrm{AD}$. The mean age at $\mathrm{A}-\mathrm{AD}$ onset varied from 15 years in patients with APS1, 35 years in those with APS2, 32 years in those with APS4, and 28 years in those with isolated A-AD.

\section{Adrenal autoimmunity}

ACA were measured in 619 patients with AD (492 with $\mathrm{A}-\mathrm{AD}$ and 127 without $\mathrm{A}-\mathrm{AD})$ and 21-OHAbs in 473 patients with $\mathrm{AD}$ ( 370 with $\mathrm{A}-\mathrm{AD}$ and 103 without A-AD). ACA were found to be positive in $398 / 492$ $(80.1 \%)$ and 21 -OHAbs in $325 / 370(87.8 \%)$ patients with A-AD of different disease duration (Fig. 3). None of the patients with other forms of $\mathrm{AD}$ were positive for ACA and/or 21-OHAbs.

ACA were tested in 463 patients with known duration of $\mathrm{A}-\mathrm{AD}$ and they were found in 246 of $290(84.8 \%)$ patients with short disease duration ( $\leq 2$ years), in 74 of $105(70.5 \%)$ with intermediate disease duration $(>2-12$ years), and in 37 of $68(54.4 \%)$ patients with a longstanding disease ( $>12$ years) (Fig. 3 ).

21-OHAbs were tested in 303 patients with known duration of A-AD and they were positive in 170 of 189 $(90 \%)$ patients with short disease duration ( $\leq 2$ years), 


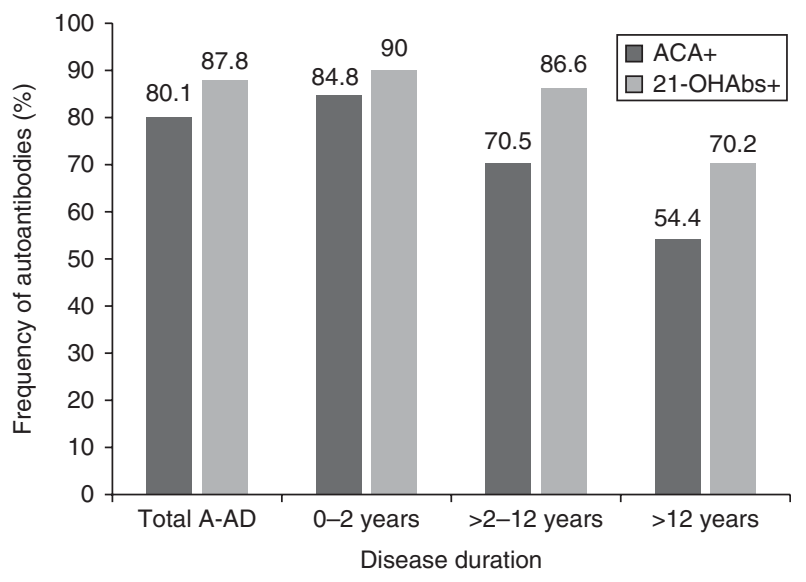

Figure 3 Prevalence of $A C A$ and 21-OHAbs in A-AD patients with different disease duration.

in 58 of $67(86.6 \%)$ with intermediate disease duration ( $>2-12$ years), and in 33 of $47(70.2 \%)$ patients with a longstanding disease ( $>12$ years) (Fig. 3).

At the onset of $\mathrm{AD}, \mathrm{ACA}$ and 21-OHAbs were respectively detected in $90.5 \%$ and in $91.7 \%$ of APS1 patients, in 88 and $93.3 \%$ of APS2 patients, in 94.7 and $100 \%$ of APS4 patients, and in 94.6 and $100 \%$ of patients with isolated A-AD.

Both ACA and 21-OHAbs were tested in the same serum sample in 326 patients with $\mathrm{AD}$ and the two tests were concordant in 278 samples $(85.3 \%)$. Particularly, $229(70.2 \%)$ were positive for both (ACA and 21-OHAbs) and $49(15 \%)$ were negative for both. There were $48(14.7 \%)$ discordant samples results: 33 (10.1\%) were ACA-negative/21-OHAbs-positive and 15 (4.6\%) were ACA-positive/21-OHAbs-negative.

\section{Nonadrenal autoimmune diseases associated with $A-A D$}

The prevalence and the associations of the major autoimmune disorders in patients with APS1 and APS2 are summarized in Table 1. The most common association in APS1 was A-AD, $\mathrm{CC}$, and $\mathrm{CH}$, while the less common was $\mathrm{A}-\mathrm{AD}$ and $\mathrm{CC}$. The most common association in APS2 was A-AD and CT, while the less common was the triad A-AD, GD, and DM1. Table 2 summarizes the prevalence of other autoimmune disorders (clinical and subclinical) and cancer in patients with APS1, APS2, APS4, and isolated A-AD observed at the end of the follow-up.

Patients with APS1 In patients with APS1, the mean age at $\mathrm{A}-\mathrm{AD}$ onset was 15 years (range 2-41). CC developed at a mean age of 8 years (range 1-58) and $\mathrm{CH}$ at a mean age of 9.6 years (range 1-51). The female:male ratio was 2.1 and adults:children ratio was 0.8 .

CT was found in 20 APS1 patients; seven patients $(35 \%)$ developed thyroiditis before the onset of A-AD, four $(20 \%)$ at the onset of $\mathrm{A}-\mathrm{AD}$, and nine $(45 \%)$ after A-AD. Only one patient had GD that developed after the onset of A-AD. In patients positive for thyroid autoantibodies alone at the onset or after the diagnosis of $\mathrm{A}-\mathrm{AD}$, the annual incidence of thyroid autoimmune diseases was 3\%/year (mean follow-up $6.8 \pm 7.1$ years). At the end of the observation period, nine patients $(12.5 \%)$ were positive only for thyroid autoantibodies and continued to be followed up (Table 2).

Pancreatic autoimmunity was evident in four patients with DM1, all diagnosed before A-AD. Twenty-six patients $(36 \%)$ were found to be positive for ICA and/or GADAbs at the onset or after the onset of A-AD, but none developed overt DM1 during follow-up (mean $10.8 \pm 8.9$ years) (Table 2 ).

Twenty-three patients with APS1 (32\%) developed autoimmune gastritis with or without pernicious anemia, $14(61 \%)$ before and nine (39\%) after the onset of A-AD. In autoantibody-positive patients, the annual incidence of autoimmune gastritis was $2.6 \% /$ year (mean follow-up $6.2 \pm 6.1$ years). At the end of the observation period, 18 patients $(25 \%)$ were positive for PCA and/or IFA and continue to be followed up (Table 2).

Celiac disease was diagnosed in one APS1 patient (1.4\%) before the onset of A-AD, while three patients had antibodies to tTG and/or endomysium (4.2\%); however, none developed disease after a mean follow-up of $7.5 \pm 9.4$ years. These patients continue to be followed up (Table 2).

Ovarian autoimmunity was found in 19 females (39\%) who developed POF after $5.8 \pm 6.9$ years from the diagnosis of A-AD. In all cases, POF was preceded by the presence of StCA and/or 17 $\alpha-\mathrm{OHAbs}$ and/or SCCAbs, and the annual incidence of POF in antibody-positive patients was $2 \%$ year. At the end of follow-up 17 (35\%) patients were StCA and/or $17 \alpha-\mathrm{OHAbs}$ and/or SCCAbs positive and continue to be monitored (Table 2).

Table 1 Prevalence and combinations of the three major autoimmune disease components in 72 patients with APS1 and in 322 patients with APS2.

\begin{tabular}{lcc}
\hline & $\begin{array}{c}\text { No. of } \\
\text { cases }\end{array}$ & $\begin{array}{c}\text { Prevalence } \\
(\%)\end{array}$ \\
\hline Main disease associations in APS1 & & \\
A-AD + chronic candidiasis + chronic & $47 / 72$ & 66.2 \\
hypoparathyroidism & & \\
A-AD + chronic hypoparathyroidism & $17 / 72$ & 23.9 \\
A-AD+ chronic candidiasis & $8 / 72$ & 9.9 \\
$\begin{array}{l}\text { Main disease associations in APS2 } \\
\text { A-AD+ chronic thyroiditis }\end{array}$ & $236 / 322$ & 73.4 \\
A-AD+ Graves' disease & $35 / 322$ & 10.8 \\
A-AD+ chronic thyroiditis + type 1 & $25 / 322$ & 7.7 \\
diabetes mellitus & & \\
A-AD+type 1 & $20 / 322$ & 6.2 \\
diabetes mellitus & $6 / 322$ & 1.9 \\
A-AD + Graves' disease + type 1 & & \\
diabetes mellitus & & \\
\hline
\end{tabular}

A-AD, autoimmune Addison's disease; APS1, autoimmune polyendocrine syndrome type 1 . 


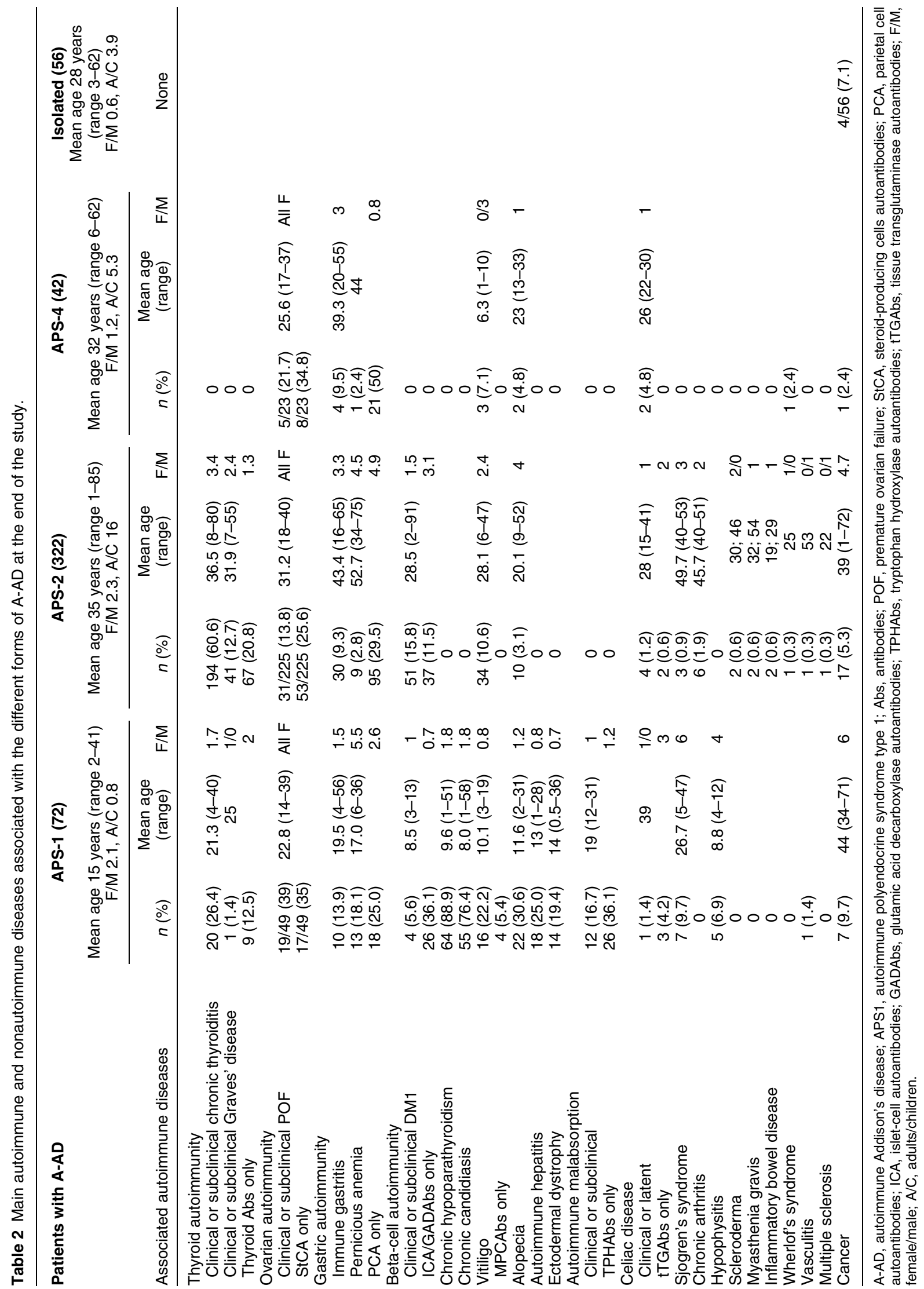


Sixteen patients $(22.2 \%)$ were affected by vitiligo and all were positive for MPCAbs. Vitiligo was evident before $\mathrm{A}-\mathrm{AD}$ in seven cases $(43.7 \%)$ at the onset of $\mathrm{A}-\mathrm{AD}$ in three $(18.8 \%)$ and after $\mathrm{A}-\mathrm{AD}$ in six $(37.5 \%)$. The annual incidence of vitiligo in MPCAbs-positive patients was $1.2 \%$ year (mean follow-up $6.4 \pm 5.5$ years). At the end of the observation period, four patients had MPCAbs and continue to be monitored (Table 2). Twenty-two patients $(30.6 \%)$ were affected by alopecia that developed before A-AD in $13(59.1 \%)$, at the onset of $\mathrm{A}-\mathrm{AD}$ in four $(18.2 \%)$, and after $\mathrm{A}-\mathrm{AD}$ in five patients (22.7\%) (Table 2). Eighteen (25\%) patients developed autoimmune hepatitis: before A-AD in eight cases $(44.4 \%)$, at the same time in three cases $(16.7 \%)$, and after $\mathrm{A}-\mathrm{AD}$ in seven cases $(38.9 \%)$. Twelve patients (16.7\%) were affected by autoimmune malabsorption with positive TPHAbs, while 26 (36\%) were found to be positive for TPHAbs and are being followed up for gastrointestinal function and serum levels of serotonin (Table 2). Seven patients (9.7\%) developed cancer of the upper digestive tract (three mouth, two esophagus, and two stomach). One of these patients developed multiple tumors.

Patients with APS2 In patients with APS2, the mean age at $\mathrm{A}-\mathrm{AD}$ onset was 34.6 years (range $1-85$ ), DM1 28.5 years (range 2-91), and autoimmune thyroid diseases 35.8 years (range $7-80$ ). The female:male ratio was 2.3 and the adult:children ratio was 16/1 (Table 2).

In this group, 235 (68.4\%) patients developed clinical or subclinical thyroid autoimmune diseases: specifically 194 had CT and 41 had GD (Table 2). Of the 194 who developed CT, the diagnosis was made before the onset of A-AD in 96 (49\%), at the onset of A-AD in 54 (28\%), and after the diagnosis of A-AD in $44(23 \%)$. Of the 41 patients with GD, the disease was diagnosed before A-AD in $29(71 \%)$, at the onset of A-AD in one $(2 \%)$, and after $\mathrm{A}-\mathrm{AD}$ in $11(27 \%)$. In patients with thyroid autoantibodies, the annual incidence of progression to clinical or subclinical thyroid disease was $8 \% /$ year (mean follow-up $3.4 \pm 5.5$ years). At the end of the follow-up, 67 patients $(20.8 \%)$ had thyroid autoantibodies with normal thyroid function and are followed up by measurement of serum TSH on an annual basis (Table 2).

Autoimmunity toward pancreatic islet cells was found in 51 patients $(15.8 \%)$ who presented with DM1. DM1 was diagnosed before A-AD in 36 (71\%), at the onset of A-AD in seven (14\%), and after A-AD in eight $(16 \%)$ patients. In subjects with ICA and/or GADAbs detected at the onset or after A-AD, DM1 developed with an annual incidence of $2 \%$ /year (mean follow-up $6.3 \pm 7.1$ years). At the end of the observation period, 37 patients $(11.5 \%)$ were ICA and/or GADAbs positive and are followed up by an annual oral glucose tolerance test (OGTT) (Table 2).

Autoimmune gastritis with or without pernicious anemia was diagnosed in $39(12 \%)$ patients, before
$\mathrm{A}-\mathrm{AD}$ in five $(12.8 \%)$, at the onset of $\mathrm{A}-\mathrm{AD}$ in five $(12.8 \%)$, and after A-AD in $29(74.4 \%)$ patients. The annual incidence of clinical or subclinical disease in patients with gastric autoantibodies was $2 \% /$ year (mean follow-up $5.4 \pm 6.0$ years). At the end of the observation period, 95 patients $(29.5 \%)$ were PCA and/or IFA positive and are followed up annually by measurement of gastrinemia and red blood cell count (Table 2).

Thirty-four patients (10.6\%) were affected by vitiligo, all being negative for MPCAbs. Vitiligo was discovered before $\mathrm{A}-\mathrm{AD}$ in $23(67.6 \%)$, at the onset of $\mathrm{A}-\mathrm{AD}$ in five $(14.7 \%)$, and after $\mathrm{A}-\mathrm{AD}$ in six patients $(17.6 \%)$ (Table 2$)$. Ten patients $(3.1 \%)$ were affected by alopecia that developed before A-AD in six $(60 \%)$, at the onset of A-AD in three $(30 \%)$, and after A-AD in one patient (10\%) (Table 2).

Celiac disease was diagnosed in four patients $(1.2 \%)$, one $(25 \%)$ before the diagnosis of $\mathrm{A}-\mathrm{AD}$, and three (75\%) after the onset of A-AD. In five patients with tTGAbs, the annual incidence of celiac disease was $30 \% /$ year (mean follow-up 3.0 \pm 4.1 years). At the end of follow-up, two patients $(0.6 \%)$ were positive for tTGAbs and continued the follow-up (Table 2).

POF was diagnosed in 31 patients (13.8\%). All developed POF after the onset of $\mathrm{A}-\mathrm{AD}$ and all were found to be positive for StCA and/or $17 \alpha-\mathrm{OHAbs}$ and/or SCCAbs before the onset of the disease. In patients positive for StCA and/or $17 \alpha-O H A b s$ and/or SCCAbs, an annual incidence of POF was found to be $2 \%$ year (mean follow-up $4.7 \pm 2.8$ years). At the end of the observation period, 53 patients were StCA and/or $17 \alpha$ OHAbs and/or SCCAbs positive and their gonadal function is followed up yearly (Table 2).

Seventeen patients (5.3\%) developed cancer: eight breast cancer, two papillary thyroid carcinoma, one medullary thyroid carcinoma, one nonsecreting pituitary adenoma, two cutaneous tumors, one astrocytoma, one meningioma, and one exocrine pancreatic carcinoma.

Patients with APS4 In patients with APS4, the mean age at A-AD onset was 32 years (range 6-62), the female:male ratio was 1.2 and adults:children ratio was 5.3.

Five patients $(11.9 \%)$ were affected by autoimmune gastritis, with or without pernicious anaemia. In four $(80 \%)$ the condition was diagnosed before and in one $(20 \%)$ after the onset of A-AD. In patients with PCA and/or IFA, the annual incidence of the disease was $3 \% /$ year.

Five females had POF $(21.7 \%)$ and in all the disease occurred after the diagnosis of A-AD. In all patients, POF was preceded by the presence of StCA and/or $17 \alpha$ $\mathrm{OHAbs}$ and/or SCCAbs with an annual incidence of $6 \% /$ year. At the end of the observation period, eight females were positive for StCA and/or $17 \alpha$-OHAbs and/or SCCAbs and their gonadal function is assessed on yearly basis. Three patients $(7.1 \%)$ were affected by 
vitiligo, all being negative for MPCAbs. In these patients, vitiligo developed before $\mathrm{A}-\mathrm{AD}$ in two and after $\mathrm{A}-\mathrm{AD}$ in one. Celiac disease developed in two patients (4.8\%); in both the disease was diagnosed after A-AD on the basis of the positive test for tTGAb. Two patients $(4.8 \%)$ were affected by alopecia and both developed it after the onset of A-AD (Table 2). One patient (2.4\%) developed cancer (lymphoblastic leukemia).

Patients with isolated $\boldsymbol{A}-\boldsymbol{A D}$ In patients with isolated $\mathrm{A}-\mathrm{AD}$, the mean age at $\mathrm{AD}$ onset was 28 years (range 3-62), the female:male ratio was 0.6 and adults:children ratio was 3.9 (Table 2). There were four cases of cancer among these patients: one had papillary thyroid carcinoma, one testicular carcinoma, one giant cell cutaneous carcinoma, and one had hip-bone osteoma.

\section{Adrenal imaging}

Radiologic imaging of adrenal glands was performed in 240 patients with A-AD. Ultrasound adrenal examination was performed in 123 patients: normal adrenal glands were found in 113 patients (92\%) while in ten patients they were not detectable $(8 \%)$. CT scan was performed in 108 and was normal in 66 subjects (61\%), in 41 patients the adrenals were reduced in size/volume and in one patient (39\%) there was a slight increase in adrenal volume. RMN examination was performed in nine subjects and was normal in four $(44 \%)$ patients, while in five $(56 \%)$ the adrenals were reduced in size/volume. None of the adrenal glands showed calcifications. There were no differences in adrenal imaging between the different forms of A-AD.

\section{HLA-DRB1 frequencies}

The frequency of class II HLA haplotypes DRB1*03 and DRB1*04 was significantly increased only in the APS2 group $(P<0.0001)$, while DRB1*01, DRB1*07, $\mathrm{DRB}^{*} 11$, and DRB1*13 were significantly reduced $(P<0.0001)$ in patients with APS2 (with or without DM1 or autoimmune thyroid diseases). In isolated $\mathrm{A}-\mathrm{AD}$, only $\mathrm{DRB} 1{ }^{*} 03$ was increased, also if not significantly different from controls $(P=0.06)$.

\section{AIRE gene mutations}

AIRE gene mutations in homozygosis or in compound heterozygosis were found in 67 of 72 patients $(93 \%)$ with A-AD in the context of APS1. In five patients, no AIRE gene mutations were detected. IFNAbs were detectable in 69 of 72 patients $(95.8 \%)$ with APS1. Two of the five patients without AIRE gene mutations were also IFNAbs negative. Out of 30 patients who developed A-AD before 20 years of age and without other major components of APS1, all being negative for IFNAbs, AIRE gene mutations were found in only one case. This was a female with $\mathrm{R} 471 \mathrm{C} / \mathrm{R} 471 \mathrm{C}$ mutation on exon 12 who developed A-AD at 14 years of age and later during follow-up (at the age of 18 years) developed vaginal candidiasis resistant to antifungal therapy, consistent with the clinical presentation of APS1.

\section{Patients with TBC-AD}

In the 57 patients with $\mathrm{TBC}-\mathrm{AD}$, the mean age at disease onset was 52 years (range 19-75). There were 15 females and 42 males with a female:male ratio of 0.36 . Figure 2 shows the age of TBC-AD onset at different decades of life. The highest number of cases was diagnosed in the fifth and sixth decades of life (36 of 57 patients, $63.2 \%$ ). The patients with TBC-AD were followed up for a mean period of 10.5 years (range $0.5-46$ years).

ACA and 21-OHAbs were negative in all the 50 tested patients with TBC-AD. Some patients had associated diseases, two patients had alopecia $(3.5 \%)$, one vitiligo (1.8\%), two DM2 (3.5\%), two DM1 (3.5\%), one pemphygus $(1.8 \%)$, and two had cancer $(3.5 \%)$ (one multiple myeloma and one papillary thyroid carcinoma).

In the case of 50 reviewed patients with $\mathrm{TBC}-\mathrm{AD}$, radiologic imaging showed an increase in adrenal volume, with or without calcifications, in 45 patients $(90 \%)$. In the remaining five patients, adrenal glands appeared small, with calcifications or fibrosis.

\section{Patients with adrenal cancer}

Out of the ten patients with C-AD, two had primary adrenal carcinoma and eight had metastatic involvement of both adrenal glands due to different cancers (four renal carcinomas, three nonHodgkin lymphoma, and one breast carcinoma). The mean age at onset of $\mathrm{C}$-AD was 55 years (range 31-78) with a female:male ratio of 2.3. All ten patients with $\mathrm{C}$-AD were negative for ACA and 21-OHAbs. On imaging, bilateral adrenal masses were evident in all the cases.

\section{Patients with genetic forms of $A D$}

Twenty-four of 29 (all males) had adrenoleukodystrophy with a mean age at onset of 18 years (range 1-63), two had 21-hydroxylase deficiency, one had X-linked congenital adrenal hypoplasia with mutation of $D A X 1$ (NROB1) (dosage-sensitive sex reversal, adrenal hypoplasia critical region, on chromosome X, gene 1), one had POEMS (polyneuropathy, organomegaly, endocrinopathy, M-protein skin abnormalities), and one had ACTH receptor mutation. ACA and 21-OHAbs were negative in 22 patients tested.

\section{Patients with AD after adrenalectomy}

This group included six patients (four developed adrenal insufficiency after bilateral adrenalectomy for Cushing's syndrome, one underwent surgery for benign bilateral 
adrenal masses, and one for a Carney complex). All six patients were negative for ACA and 21-OHAbs.

\section{Patients with vascular disorders}

In four cases, adrenal failure was due to vascular disorders: two had anti-phospholipid syndrome with imaging showing increased adrenal volume with bilateral hemorrhagic infarction, one had congenital bilateral hemorrhage, and one had WaterhouseFriderichsen syndrome. All these patients were negative for ACA and/or 21-OHAbs. Imaging in patients with anti-phospholipid syndrome revealed bilateral increased adrenal volume with a central area of hemorrhage.

\section{Patients with rare infections}

In three patients, adrenal failure appeared after infections involving the adrenals: two had blastomycosis and one had HIV. All these three patients were negative for ACA and 21-OHAbs.

\section{Treatment of patients with AD}

Five-hundred and thirty-five patients with $\mathrm{AD}$ were on cortisone acetate (mean dose $31.5 \mathrm{mg}$ /daily, range 25$50 \mathrm{mg} /$ daily) and only 75 were on hydrocortisone (mean $15 \mathrm{mg} /$ daily, range 10-30 mg/daily). The medications were usually taken in two or three doses/day. A large proportion of the patients were also on fludrocortisone treatment (mean dose $0.075 \mu \mathrm{g} /$ daily, range $0.05-0.2 \mu \mathrm{g} /$ day) in one morning dose. Patients were also receiving appropriate treatment for the associated diseases, when present.

\section{Discussion}

We reviewed 633 patients with $\mathrm{AD}$ registered at the Endocrine Unit in Padova during the years 1967-2010. This represents the largest cohort of patients with $\mathrm{AD}$ from one national region described to date. The great majority of patients $(77.7 \%)$ had $A-A D$, while $9 \%$ had TBC-AD. Patients with other forms of AD (genetic, postsurgical, vascular, or infectious) were the minority of the cases $(8 \%)$, and in only $5 \%$ the etiological diagnosis was missing (idiopathic forms). Evaluating how different forms of $\mathrm{AD}$ varied in diagnosis frequency during the years, as expected, we found a progressive increase in autoimmune forms from 54.2 to $83.7 \%$, while TBC-AD decreased from 33.3 to $3 \%$. The other forms remained stable during the observation period (12.5-13.3\%).

The autoimmune nature of $\mathrm{AD}$ was denoted by the presence of ACA and/or 21-OHAbs. When ACA and/or 21-OH Abs were tested within 2 years from the diagnosis of adrenal insufficiency, more than $90 \%$ of the patients were positive; however, autoantibody positivity decreased over time after the onset. The prevalence of ACA/21-OHAbs among patients with different clinical presentations of $\mathrm{A}-\mathrm{AD}$, i.e. isolated or in the context of an APS, was not significantly different.

There was an association between ACA and/or 21-OHAbs positivity and adrenal imaging findings. All adrenal antibody-positive patients had normal or atrophic glands on imaging and only one of the 240 patients examined showed a slight increase in adrenal volume, probably related to inflammatory infiltration of the glands. This is consistent with the observations that detection of ACA/21-OHAbs is the test of choice for identifying the autoimmune nature of $\mathrm{AD}$ while adrenal imaging provides no additional value for the diagnosis (13). For these reasons, adrenal imaging was no longer performed in ACA/21-OHAbs-positive patients recruited more recently in our study. However, adrenal imaging may be necessary in clarifying the nature of $\mathrm{AD}$ in adrenal antibody-negative patients (13).

Many studies reported that clinically associated autoimmune manifestations were present in no more than $50 \%$ of A-AD patients $(1,19)$. However, according to more recent criteria, the natural history of autoimmune endocrine disorders is usually characterized by three sequential stages, i.e. potential, subclinical, and clinical disease $(1,23)$. Consequently, patients with APS may present two or three clinical diseases or at least either one clinical disease and another subclinical manifestation or one clinical disease with autoantibody positivity only. According to these criteria, the authors of a recent nationwide Norwegian study demonstrated that the association with a clinical or subclinical autoimmune diseases, or autoantibody positivity only, was found in $88 \%$ of patients with A-AD, while only $12 \%$ had isolated A-AD (8).

In the present survey, after a mean observation period of 10 years, $88.6 \%$ of $\mathrm{A}-\mathrm{AD}$ patients were presented with at least one or more clinical or subclinical autoimmune disorder or autoantibody positivity only, while only $11.4 \%$ of patients were free from any autoimmune manifestations, and therefore had isolated A-AD. Our data confirm the results of the Norwegian study on $\mathrm{A}-\mathrm{AD}$ in Italy.

Considering the various autoimmune combinations, APS2 was the most common form and was found in $65.6 \%$ of patients with A-AD, followed by APS1 diagnosed in $14.4 \%$ and APS4 found in $8.5 \%$. These observations confirm that $\mathrm{A}-\mathrm{AD}$ is a condition that shows the highest association with other autoimmune diseases. Previously, we have reported a prevalence of $5 \%$ of celiac disease in 109 patients with A-AD; however, in the current study the prevalence of clinical or latent celiac disease was $1.6 \%$ (34).

In this study, we have assessed for the first time the different timing of development of other autoimmune disorders in relation to the onset of A-AD. Furthermore, we have calculated the risk of developing new clinical or subclinical diseases in patients with other organ-specific autoantibodies only, expressed as annual incidence. We 
have found that both the timing of development and the annual incidence of other autoimmune diseases varied greatly in different forms of A-AD. For example, the presence of thyroid antibodies was associated with an almost threefold greater risk of developing thyroid diseases in patients with APS2 than in those with APS1 (8 vs 3\%/year respectively). In addition, 3/5 APS2 patients positive for tTGAbs developed celiac disease while none of tTGAbs-positive APS1 patients progressed to overt disease. The number of patients who progressed to overt celiac disease was low and this limited the calculation of the risk of developing the disease. However, these observations suggest that the risk of progression to overt thyroid or celiac diseases may vary greatly depending on different APS type.

The prevalence of class II HLA alleles DRB1*03 and DRB1*04 was significantly increased, while that of DRB1*01, DRB1*07, DRB1*013 was reduced in patients with APS2 only compared with controls, confirming previous reports (8). Regarding other A-AD forms, isolated $\mathrm{A}-\mathrm{AD}$ showed an increment of $\mathrm{DRB} 1{ }^{*} 03$ near statistical significance, but the small number of patients studied in this group may be a limiting factor. Almost all patients with APS1 in our survey (96\%) had AIRE gene mutations, in agreement with previous studies $(33,35$, $36,37,38)$; no correlation with class II HLA alleles was detected in this group.

Analysis of patients with different forms of $A-A D$ showed common and different features. Common features were positivity for adrenal cortex antibodies and imaging of adrenal glands. Different features included the patients' age at disease onset (younger in APS1), the female:male ratio (higher in APS1 and APS2 compared with other forms), the presence of AIRE gene mutations and IFNAbs (in patients with APS1), the association with specific class II HLA antigens (in patients with APS2), as well as differences in the prevalence and annual incidence of other autoimmune diseases and cancer (Table 3).

In our patients, the second most frequent cause of adrenal failure was tuberculosis. In contrast to A-AD, $\mathrm{TBC}-\mathrm{AD}$ developed later in life, was more prevalent in males than females and diagnosed only in adults. ACA and/or 21-OHAbs were typically absent, while imaging showed enlarged adrenal glands with or without calcifications. These features clearly discriminate TBC-AD from A-AD (Table 3). The other forms of $\mathrm{AD}$ had genetic, vascular, post-surgical, malignant, or infectious origin.

The analysis of the distinctive features of $\mathrm{AD}$ reported in this study should be helpful in identifying the various etiological forms of $\mathrm{AD}$, as well as classifying the different forms of A-AD. A-AD was found to be the predominant form of adrenal insufficiency in Italy and ACA/21-OHAbs are excellent serological markers of the disease, being usually detectable in more than $90 \%$ of the patients at the onset of the disease.

The outcome of this study would be a recommendation that in order to optimize health care costs and improve the diagnostic work-up of AD, ACA or 21-OHAbs tests should be carried out first preferably at

Table 3 Main features of patients with A-AD and TBC-AD.

\begin{tabular}{|c|c|c|c|c|c|}
\hline & \multicolumn{5}{|c|}{ Etiological forms } \\
\hline & \multicolumn{4}{|c|}{ A-AD $(n=492 ;$ Frequency $77.7 \%)$} & \multirow[t]{2}{*}{ TBC-AD $(n=57)$} \\
\hline & APS-1 & APS-2 & APS-4 & Isolated & \\
\hline Frequency & $14.4 \%$ & $65.6 \%$ & $8.5 \%$ & $11.4 \%$ & $9 \%$ \\
\hline Female:male ratio & 2.1 & 2.3 & 1 & 0.6 & 0.4 \\
\hline Adults:children ratio & 0.8 & 16 & 5.3 & 3.9 & 57 \\
\hline Mean age at AD onset (years) & 15 & 35 & 32 & 28 & 52 \\
\hline Range & $2-41$ & $1-85$ & $6-62$ & $3-62$ & $19-75$ \\
\hline Family history for & $\begin{array}{c}\text { Autoimmune } \\
\text { diseases }\end{array}$ & $\begin{array}{c}\text { Autoimmune } \\
\text { diseases }\end{array}$ & $\begin{array}{c}\text { Autoimmune } \\
\text { diseases }\end{array}$ & $\begin{array}{c}\text { Autoimmune } \\
\text { diseases }\end{array}$ & TBC \\
\hline Class II HLA & No & $\begin{array}{l}\text { DRB } 1{ }^{*} 03 \\
\text { DRB } 1{ }^{*} 04\end{array}$ & $?$ & DRB $1^{*} 03$ & NT \\
\hline AIRE gene mutations & Present & Absent & Absent & Absent & Absent \\
\hline Chronic candidiasis & $76 \%$ & Absent & Absent & Absent & Rare \\
\hline Chronic hypoparathyroidism & $90 \%$ & Absent & Absent & Absent & \\
\hline Thyroid autoimmune diseases & $41 \%$ & $93.8 \%$ & Absent & Absent & \\
\hline Type 1 diabetes mellitus & $6 \%$ & $15.5 \%$ & Absent & Absent & \\
\hline Other autoimmune diseases & Up to $57 \%$ & Up to $43 \%$ & $100 \%$ & Absent & Up to $7 \%$ \\
\hline Ectodermal dystrophy & Present & Absent & Absent & Absent & Absent \\
\hline Cancer & $9.9 \%$ & $5.3 \%$ & $2.6 \%$ & $7.1 \%$ & $4 \%$ \\
\hline ACA at onset & $90.5 \%$ & $88.0 \%$ & $94.7 \%$ & $94.6 \%$ & $0 \%$ \\
\hline 21-OHAbs at onset & $91.7 \%$ & $93.3 \%$ & $100.0 \%$ & $100 \%$ & $0 \%$ \\
\hline IFN $\omega$ Abs & Present & Absent & Absent & Absent & Absent \\
\hline Imaging of adrenals & Normal/atrophic & Normal/atrophic & Normal/atrophic & Normal/atrophic & $\begin{array}{l}\text { Enlarged with } \\
\text { calcifications }\end{array}$ \\
\hline
\end{tabular}

A-AD, autoimmune Addison's disease; APS1, autoimmune polyendocrine syndrome type 1; TBC-AD, tuberculous AD; ACA, adrenal cortex autoantibodies; 21-OHAbs, 21-hydroxylase autoantibodies; NT, not tested; IFNAbs, antibodies to interferon; NMR, nuclear magnetic resonance; CT, computed tomography. 
the diagnosis of adrenal insufficiency. The 21-OHAbs test performed by RIA is more sensitive but may be more expensive than ACA by immunofluorescence. In the case of detectable adrenal autoantibodies, A-AD could be diagnosed with confidence and adrenal imaging would not be necessary. In contrast, adrenal imaging and further investigations would be indicated in adrenal antibody-negative patients. However, patients with A-AD should be screened for nonadrenal autoantibodies (at least to thyroid, parietal cell, intrinsic factor, islet cell, or GAD and, in females, to StCA or SCC or $17 \alpha \mathrm{OH}$ ) as these could be serological markers of a subclinical dysfunction of the target organ(s). Furthermore, autoantibody-positive patients with normal function of the target organ should be followed up. In addition, in the case of family history of autoimmune diseases, specific autoantibodies and biochemical/function tests should be carried out, in particular when a clinical disease is suspected.

This approach should allow the clinicians to identify A-AD patients with polyendocrine autoimmune involvement at a very early stage and alert to a possibility of the development of associated diseases. Furthermore, when required, appropriate treatment interventions could be commenced without delay.

\section{Declaration of interest}

S Chen, B Rees Smith, and J Furmaniak are employed by RSR Ltd. RSR Ltd. is a developer of medical diagnostics including kits for measuring 21-OH autoantibodies.

\section{Funding}

This study was supported in part by a grant from the European Union Seventh Framework Program, the Euradrenal project: Pathophysiology and Natural Course of Autoimmune Adrenal Failure in Europe. Grant Agreement No. 2008-201167; and from Grant ex 60\% of University of Padova.

\section{References}

1 Betterle C, Dal Pra C, Mantero F \& Zanchetta R. Autoimmune adrenal insufficiency and autoimmune polyendocrine syndromes: autoantibodies, autoantigens and their applicability in diagnosis and disease prediction. Endocrine Reviews 200223 327-364. (doi:10.1210/er.23.3.327)

2 Mason AS, Meade TW, Lee JA \& Morris JN. Epidemiological and clinical picture of Addison's disease. Lancet $19682744-747$. (doi:10.1016/S0140-6736(68)90948-3)

3 Nerup J. Addison's disease-clinical studies. A report of 108 cases. Acta Endocrinologica 197476 121-141.

4 Kong MF \& Jeffocoate W. Eighty-six cases of Addison's disease. Clinical Endocrinology 199441 757-761. (doi:10.1111/j.13652265.1994.tb02790.x)

5 Willis AC \& Vince FP. The prevalence of Addison's disease in Coventry, UK. Postgraduate Medical Journal 199773 286-288. (doi:10.1136/pgmj.73.859.286)

6 Laureti S, Vecchi L, Santeusanio F \& Falorni A. Is the prevalence of Addison's disease understimated? Journal of Clinical Endocrinology and Metabolism 199984 1762. (doi:10.1210/jc.84.5.1762)
7 Løvås K \& Husebye ES. High prevalence and increasing incidence of Addison's disease in western Norway. Clinical Endocrinology 2002 56 787-791. (doi:10.1046/j.1365-2265.2002.t01-1-01552.x)

8 Erichsen MM, Lovas K, Skinningsrud B, Wolff AB, Undlien DE, Svartberg J, Fougner KJ, Berg TJ, Bollerslev J, Mella B et al. Clinical, immunological, and genetic features of autoimmune primary adrenal insufficiency: observation from Norvegian Registry. Journal of Clinical Endocrinology and Metabolism 200994 1-9. (doi:10.1210/jc.2009-1368)

9 Jacobson DL, Gange SJ, Rose NR \& Graham NM. Epidemiology and estimated population burden of selected autoimmune diseases in the United States. Clinical Immunology and Immunopathology 1997 84 223-243. (doi:10.1006/clin.1997.4412)

10 Guttman PH. Addison's disease: statistical analysis of 566 cases and study of the pathology. Archives of Pathology 193010 $742-895$.

11 Dunlop D. Eighty-six cases of Addison's disease. BMJ 19633 887-891. (doi:10.1136/bmj.2.5362.887)

12 Laureti S, Aubourg P, Calcinaro F, Rocchiccioli F, Casucci G, Angeletti G, Brunetti P, Lernmark A, Santeusanio F \& Falorni A. Etiological diagnosis of primary adrenal insufficiency using an original flow chart of immune and biochemical markers. Journal of Clinical Endocrinology and Metabolism $1998 \quad 83$ 3163-3168. (doi:10.1210/jc.83.9.3163)

13 Falorni A, Laureti S, De Bellis AM, Zanchetta R, Tiberti C, Arnaldi G, Bini V, Beck-Peccoz P, Bizzarro A, Dotta F et al. Italian Addison network study: update of diagnostic criteria for the etiological classification of primary adrenal insufficiency. Journal of Clinical Endocrinology and Metabolism 200489 1598-1604. (doi:10.1210/jc.2003-030954)

14 Fichna M, Fichna P, Gryczynska M, Walkowiak J, Zurawek M \& Sowiński J. Screening for associated autoimmune disorders in Polish patients with Addison's disease. Endocrinology 201037 349-360. (doi:10.1007/s12020-010-9312-x)

15 Kasperlik-Zaluska AA, Czarnocka B, Jeske W \& Papierska L. Addison's disease revisited in Poland: year 2008 versus 1990. Autoimmune Diseases 20102010 731834. (doi:10.4061/2010/ 731834)

16 Ten S, New M \& Maclaren N. Addison's disease 2001. Journal of Clinical Endocrinology and Metabolism 200186 2009-2022. (doi:10.1210/jc.86.7.2909)

17 Perry R, Kecha O, Paquette J, Vliet GV \& Deal C. Primary adrenal insufficiency in children: twenty years experience at the SainteJustine Hospital, Montreal. Journal of Clinical Endocrinology and Metabolism 200590 3243-3250. (doi:10.1210/jc.2004-0016)

18 Hsieh S \& White PC. Presentation of primary adrenal insufficiency in childhood. Journal of Clinical Endocrinology and Metabolism 2011 96 E925-E928. (doi:10.1210/jc.2011-0015)

19 Leelarathna L, Breen L, Powrie JK, Thomas SM, Guzder R, McGowan B \& Carroll PV. Comorbidities, management and clinical outcome of autoimmune Addison's disease. Endocrinology 2010 38 113-117. (doi:10.1007/s12020-010-9359-8)

20 Troncone R \& Jabri B. Celiac disease and gluten sensitivity. Journal of Internal Medicine 2011269 582-590. (doi:10.1111/ j.1365-2796.2011.02385.x)

21 Scarpa R, Alaggio R, Norberto L, Furmaniak J, Chen S, Smith BR, Masiero S, Morlin L, Plebani M, De Luca F et al. Tryptophan hydroxylase autoantibodies as markers of a distinct autoimmune gastrointestinal component of autoimmune polyendocrine syndrome type 1. Journal of Clinical Endocrinology and Metabolism 201398 704-712. (doi:10.1210/jc.2012-2734)

22 Neufeld M \& Blizzard RM. Polyglandular autoimmune diseases. In Symposium on Autoimmune Aspects of Endocrine Disorders, pp 357365. Eds A Pinchera, D Doniach, GF Fenzi \& L Baschieri, New York: Academic Press, 1980.

23 Betterle C \& Presotto F. Autoimmune polyendocrine syndromes (APS) or multiple autoimmune syndromes (MAS). In Handbook of Systemic Autoimmune Diseases, Volume 9. Endocrine Manifestations of Systemic Autoimmune Diseases, pp 135-148. Eds S Walker \& LJ Jara, Amsterdam: Elsevier BV, 2008. 
24 Tanaka H, Perez MS, Powell M, Sandres JF, Sawicka J, Chen S, Prentice L, Asawa T, Betterle C, Volpato M et al. Steroid 21-hydroxylase autoantibodies: measurements with a new immunoprecipitation assay. Journal of Clinical Endocrinology and Metabolism 199782 1440-1446. (doi:10.1210/jc.82.5.1440)

25 Betterle C, Volpato M, Pedini B, Chen S, Smith BR \& Furmaniak J. Adrenal-cortex autoantibodies and steroid producing cells autoantibodies in patients with Addison's disease: comparison of immunofluorescence and immunoprecipitation assays. Journal of Clinical Endocrinology and Metabolism $1999 \quad \mathbf{8 4} \quad 618-622$. (doi:10.1210/jc.84.2.618)

26 Falorni A, Chen S, Zanchetta R, Yu L, Tiberti C, Bacosi ML, Furmaniak J, Bini V, Dotta F, Eisenbarth GS et al. Measuring adrenal autoantibody response: inter laboratory concordance in the first international serum exchange for the determination of 21-hydroxylase autoantibodies. Clinical Immunology 2011140 291-299. (doi:10.1016/j.clim.2011.04.012)

27 Betterle C, Greggio NA \& Volpato M. Autoimmune polyglandular disease type 1. Journal of Clinical Endocrinology and Metabolism 199883 1049-1055. (doi:10.1210/jc.83.4.1049)

28 Chen S, Sawicka J, Betterle C, Powell M, Prentice L, Volpato M, Rees Smith B \& Furmaniak J. Autoantibodies to steroidogenic enzymes in autoimmune polyglandular syndrome, Addison's disease, and premature ovarian failure. Journal of Clinical Endocrinology and Metabolism 199683 2977-2986. (doi:10.1210/jc.83.8.2977)

29 Basso D, Guariso G, Fasolo M, Pittoni M, Schiavon S, Fogar P, Greco E, Naviglia F, Zambon CF \& Plebani M. A new indirect chemiluminescent immunoassay to measure anti-tissue tranglutaminase antibodies. Journal of Pediatric Gastroenterology and Nutrition $2006 \quad 43$ 613-618. (doi:10.1097/01.mpg.0000 239739.09983.38)

30 Dal Pra C, Chen S, Betterle C, Zanchetta R, McGrath V, Furmaniak J \& Rees Smith B. Autoantibodies to human tryptophan hydroxylase and aromatic L-amino acid decarboxylase. European Journal of Endocrinology 2004150 313-321. (doi:10.1530/eje.0.1500313)

31 Cervato S, Morlin L, Albergoni MP, Masiero S, Greggio N, Meossi C, Chen S, del Pilar Larosa M, Furmaniak J, Rees Smith B et al. AIRE gene mutations and autoantibodies to interferon omega in patients with chronic hypoparathyroidism without APECED. Clinical Endocrinology 201073 630-636. (doi:10.1111/j.13652265.2010.03862.x)
32 Olerup O \& Zetterguist H. DR "low resolution" PCR-SSP typing: a correction and an update. Tissue Antigens 199341 55-56. (doi:10.1111/j.1399-0039.1993.tb01991.x)

33 Cervato S, Mariniello B, Lazzarotto F, Morlin L, Zanchetta R, Radetti G, De Luca F, Valenzise M. Giordano R, Rizzo D et al. Evaluation of the autoimmune regulator (AIRE) gene mutations in a cohort of Italian patients with autoimmune-polyendocrinopathy-candidiasis-ectodermal-dystrophy (APECED) and in their relatives. Clinical Endocrinology 200970 421-428. (doi:10.1111/ j.1365-2265.2008.03318.x)

34 Betterle C, Lazzarotto F, Spadaccino AC, Basso D, Plebani M, Pedini B, Chiarelli S \& Albergoni M. Celiac disease in North Italian patients with autoimmune Addison's disease. European Journal of Endocrinology 2006154 275-279. (doi:10.1530/eje.1.02089)

35 Giordano C, Modica R, Allotta ML, Guarnotta V, Cervato S, Masiero S, Giordano R, Garelli S \& Betterle C. Autoimmune polyendocrinopathy-candidiasis-ectodermal-dystrophy (APECED) in Sicily: confirmation that R203X is the peculiar AIRE gene mutation. Journal of Endocrinological Investigation 201235 384-388. (doi:10.3275/7965)

36 Betterle C, Ghizzoni L, Cassio A, Baronio F, Cervato S, Garelli S, Barbi E \& Tonini G. Autoimmune-polyendocrinopathy-candidiasisectodermal-dystrophy (APECED), in Calabria: clinical, immunological and genetic patterns. Journal of Endocrinological Investigation 201235 877-881. (doi:10.3275/8109)

37 Capalbo D, Mazza C, Giordano R, Improda N, Arvat E, Cervato S, Morlin L, Pignata C, Betterle C \& Salerno M. Molecular background and genotype-phenotype correlation in APECED patients from Campania and in their relatives. Journal of Endocrinological Investigation 201235 169-173. (doi:10.3275/7677)

38 Valenzise M, Wasniewska M, Mirabelli S, De Luca F, Cervato S \& Betterle C. Identification of two novel mutations in the first Sicilian APECED patient with no R203X mutation in AIRE gene and review of Italian APECED genotypes. Gene 2012499 343-346. (doi:10.1016/j.gene.2012.03.032)

Received 27 June 2013

Revised version received 4 September 2013

Accepted 5 September 2013 